\title{
$\Omega$
}

Geert M. Verleden ${ }^{1,2}$

[1]

'Secretary of Assembly 8, European Respiratory Society.

2University Hospital Gasthuisberg, Dept Respiratory Disease, Lung Transplantation Unit,

Leuven, Belgium.

\section{Assembly 8: where surgeons and pulmonologists meet}

\section{Meet the Assemblies}

Cite as: Verleden GM. Assembly 8: where surgeons and pulmonologists meet. Breathe 2016; 12: 187-188.
Assembly 8 represents a small, but very important assembly in the European Respiratory Society (ERS). It is the home of thoracic/cardiothoracic surgeons (group 8.1) and of the lung transplantation group (group 8.2). Although small, we represent a definite need within the society as surgery, and lung transplantation more specifically, are key elements in treatment when other options fail.

Every year we try to build interesting sessions at the ERS International Congress, liaising with other assemblies on topics such as clinical problems, thoracic oncology, respiratory infections, etc.

Over the past few years the number of members of our assembly has increased, reaching a total of 224 in 2015 (an increase of $66 \%$ compared with 2012). For the 2015 Congress in Amsterdam, 130 abstracts were received from Assembly 8 which, in absolute numbers, is the lowest among the assemblies; however, if we consider the number of accepted abstracts per assembly member, Assembly 8 ranks number six out of 11, providing a much better representation of our scientific activity.

Every year, several symposia from thoracic surgery as well as the lung transplantation group are proposed and accepted, and we like to consult our members to ensure these are highly scientific proposals. The symposia are put together based on surveys of the members of Assembly 8 and always appeal to a lot of interested delegates, although they might be quite specialist symposia, especially when they are dedicated to lung transplantation. Therefore, we try to also include at least one general symposium on lung transplantation, which may be dedicated to selection of patients, outcomes of transplantation for several underlying diseases, etc. This seems to work better than it used to, when the symposia might not have been appealing to the general pulmonologist as they were dealing with very specific topics.

The same holds true for thoracic surgery, where the questions of pulmonologists regarding surgical treatment of, for instance, oncological or infectious conditions are covered. Also, with the widespread use of video-assisted techniques, the respiratory community should be kept informed about proper patient selection for these procedures, which aims to determine their proper role in respiratory medicine.

Besides symposia, we offer abstract sessions and oral presentations at the ERS International Congress, which are always very well attended. Postgraduate symposia on thoracic surgery topics are presented every year, as well as meet the professor seminars. 
This range of activity clearly illustrates the need for our assembly to remain independent, as we offer a home for surgeons and some very specialised pulmonologists, who may distribute their knowledge and so contribute to the general educational need of the society.

Our assembly will continue to grow, as there is increasing interest from Eastern European countries not only to hone the skills of their thoracic and cardiothoracic surgeons and to provide interaction with pulmonologists, but also to start lung transplant programmes in these countries. Therefore, our assembly offers help in starting such programmes. Every 3 years, to date, we have organised an ERS lung transplantation course, with an eminent faculty of surgeons and pulmonary transplant physicians to provide a unique overview of how such a programme should be run and how problems may be covered. The most recent course, which was the fourth to be organised, was held in Istanbul in February 2016 and was again very successful with 65 participants from all over the world.

Therefore, Assembly 8 will always be the place where surgeons and pulmonologists meet, discuss, interact and share their views and thoughts. 